\title{
Discussions about Limiting Treatment in a Geriatric Clinic
}

\author{
Susan Dorr Goold, MD, MHSA, * Robert M. Arnold, MD, $\uparrow$ and Laura A. Siminoff, PhD $\ddagger$
}

Objective: Obtain detailed information about the frequency and content of discussions about withholding treatment between doctors and elderly outpatients.

Design: Survey.

Setting: Primary care geriatric clinic at an urban university. Participants: Twelve physicians and one nurse practitioner completed questionnaires for $185 / 198(93.4 \%)$ patient visits. Measurements: Questionnaires were completed by physicians after each patient visit during August 1989. Interviews were conducted with physicians who had discussed limiting life-sustaining treatment with patients.

Results: Ten percent $(n=19)$ of patients seen had had discussions with their physicians about life-sustaining treatment. These patients were older and had worse prognoses as estimated by their physicians. Physicians usually raised the issue with the families of demented patients and mentioned dementia, quality of life, prognosis, and the need to make other clinical decisions as motivation for initiating discussions. The majority of patients with poor prognoses, however, had not had discussions about life support.

Conclusions: Despite increasing attention given to end-oflife decisions in the medical and lay press, discussions with elderly outpatients about limiting treatment occur rarely. They are more likely when patients are older or have worse prognoses, but age, prognosis, and poor quality of life do not consistently lead physicians to raise the issue. J Am Geriatr Soc 41:277-281, 1993

ing for the critically ill. Although there is some disagreement about whether advance directives should be discussed with all patients, most medical ethicists and physicians agree that the elderly should be asked early about their preferences regarding life-sustaining treatment.

Empirical data indicate that the majority of patients wish to be involved in these decisions and that many have strong feelings about the use of life-sustaining treatments. A study of elderly outpatients reported that $87 \%$ thought that physicians should discuss CPR with their patients, and $70 \%$ thought that these discussions ought to take place before the patient became ill. ${ }^{4}$ Other studies conducted among the elderly, AIDS patients, and patients surviving resuscitation have shown a similar proclivity toward discussing these issues with their doctors. $^{5-10}$ Interestingly, although patients want to discuss these issues, studies indicate that most patients have not done so and would prefer their doctor, not they themselves, raise the issue. ${ }^{11}$ This is consistent with findings that patients are, in general, hesitant to raise issues with their physicians on their own. ${ }^{12}$

Unfortunately, studies of advance directives reveal that physicians rarely discuss these issues with their patients. In a study of elderly outpatients (excluding those with impaired cognition) Shmerling found that only $3 \%$ of patients had previously had discussions about limiting therapy with their physicians. Discussions between patients' families and physicians occurred in an additional $12 \%$. $^{4}$

We have little detailed information about physicians' behavior and motivation regarding advance directives. Few studies have closely examined physician-patient discussions about advance directives in an outpatient setting. Specifically, what factors lead physicians to raise this issue, and why? Are they uncomfortable

From the *Department of Medicine, Division of General Medicine, University of Michigan Medical Center, Ann Arbor, Michigan; and the †Division of General Medicine, and the †Department of Psychiatry, University of Pittsburgh, Pittsburgh, Pennsylvania.

Presented to the Society for General Internal Medicine, Midwest Region, November 1991.

Address correspondence to Susan Dorr Goold, MD, Division of General Medicine, Dept. of Medicine, University of Michigan Medical Center, 3116 Taubman Center Box 0376, Ann Arbor, MI 48109-0376. 
doing so? What is said? In an effort to identify patient and physician characteristics that affect discussions about limiting treatment and to gain information about the frequency and content of the discussions themselves, we surveyed, then interviewed, physicians in an outpatient geriatric clinic.

\section{METHODS}

The study sample is a case series of all patients seen at an outpatient geriatric clinic. During August 1989, all patients attending a geriatric clinic affiliated with an urban university hospital were entered into the study. This clinic is staffed by academic internists and geriatricians, residents in internal medicine, and fellows in geriatrics.

Data on all patient cases were obtained through brief questionnaires completed by the patient's physician immediately after each patient visit. A research assistant was present in the clinic and responsible for distributing and collecting the questionnaires. The instrument was designed to elicit information regarding the following: patient demographic variables, duration of physician-patient relationship, the physician's estimate of the patient's mortality and quality of life at 1 and 5 years, physician's estimate of the patient's prognosis after cardiopulmonary resuscitation (CPR), and whether the physician had ever discussed experimental protocols, financial matters, nursing home placement, hospice care, autopsy, CPR, or withholding life-sustaining therapy with the patient. Visual analog scales $(100 \mathrm{~mm})$ were used for questions requiring the physician to estimate prognosis. These scales have been found to be valid and reliable in studies examining levels of pain as reported by cancer patients and estimates of prognoses reported by physicians for individual patients. 13,14

Once the questionnaires were completed, the population was divided into discussion (case) and nondiscussion (control) patients. Discussion cases were defined as patients with whom physicians had, at some time, discussed withholding CPR or other life-sustaining treatment. All other patients served as controls.

Taped interviews with physicians were conducted about all discussion cases, using a semi-structured open-ended format. The goal of the interview was to identify who raised the issue of advance directives, to elucidate factors which led the physician to bring up the issue, and to characterize the content of the discussion and the patient's decision. An interview guide was used but interviewees were encouraged to talk freely about the issues of importance to them. One author (RMA) developed a coding scheme using content analysis of the taped interviews. For example, responses by interviewed physicians to a question about the reason for broaching the subject of life-sustaining treatment were listed, then categorized as quality-oflife, precipitating treatment decision, etc. Interviews were then coded by two separate investigators (RMA and SDG). Interrater reliability ranged from $75 \%-$ $100 \%$ for the initial coding. Investigators discussed instances of divergent coding and reevaluated those interviews. A consensus was achieved in all cases.
Data were analyzed using Student's unpaired $t$ tests for interval level data and Chi Square tests for categorical data.

This study was approved by the Institutional Review Board of University of Pittsburgh Health Center.

\section{RESULTS}

Twelve physicians and one nurse practitioner worked at the clinic during the study period. Four geriatricians and one internist treated the majority of patients $(70 \%)$. The remaining physicians included five internal medicine residents and two geriatric fellows. There were 198 unique patient visits during the month; all had questionnaires distributed to the relevant physician. Of these, 185 surveys were completed (response rate $=93.4 \%$ ).

Two-thirds of subjects were between 70 and 85 years old; $8 \%$ were younger than 65 years. Thirty-five percent were married, and $43 \%$ were widowed. Ninety percent lived at home with the remainder living in nursing homes or personal care homes. The vast majority $(89 \%)$ of physicians considered themselves the patient's primary caregiver, $57 \%$ having cared for the patient for 1 year or longer. Fifty-eight percent of all patients were judged by their physicians to be competent to make decisions regarding their care (greater than $80 \mathrm{~mm}$ on a $100 \mathrm{~mm}$ analog scale).

There were 19 patients (cases) with whom CPR or other life-sustaining treatment had been discussed; 166 patients (controls) had never had such a discussion with their physicians. In addition, of the 185 patients with completed questionnaires, $49.2 \%(n=91)$ reported discussions about prognosis, $24 \%(n=45)$ reported discussions about nursing home placement, and $1 \%(n=2)$ reported discussing autopsy. Of the 19 (10\%) cases reporting discussions about CPR, approximately half had also discussed withholding other lifesustaining treatment, and a few had discussed hospice care. An examination of patient demographics revealed no significant differences in gender, marital status, or residence between case and control patients. The number of discussions per individual physician ranged from none to seven for the month of the study.

We examined physician assessment of prognosis, hypothesizing that a worse prognosis might prompt more frequent discussions about life-sustaining treatment. Indeed, we found that discussions of CPR were associated with a worse 1 - and 5-year prognosis $(P<$ 0.05 for both), as estimated by their physicians. Despite this association, there remained 15 patients with an estimated 1-year prognosis of less than $50 \%$ who had never had a discussion with their physician about lifesustaining treatment. Cases were also, on average, older than control patients $(P=0.015)$. There were no statistically significant differences in gender or physician estimates of prognosis after CPR, quality of life, or patient competence between cases and controls (see Table 1). In fact, 23 patients $(12.4 \%)$ believed by their physicians to have a poor quality of life (less than 41 $\mathrm{mm}$ on the $1-100 \mathrm{~mm}$ analog scale) had never had a discussion with their physician about limiting treatment. 
TABLE 1. PHYSICIAN ESTIMATES OF PATIENT CHARACTERISTICS: DIFFERENCES BETWEEN DISCUSSION CASES AND NON-DISCUSSION CONTROLS

\begin{tabular}{lccc}
\hline \multicolumn{1}{c}{ Variable } & $\begin{array}{c}\text { Mean Difference } \\
\text { (Control-Case) }\end{array}$ & $\boldsymbol{t}$ & $\boldsymbol{P}$-value \\
\hline One-year prognosis & 16.263 & 2.795 & .012 \\
Five-year prognosis & 27.368 & 2.875 & .0101 \\
Competence & 16.737 & 1.309 & .2069 \\
Quality-of-Life & 8.579 & 1.103 & .2846 \\
Probability of surviving & 0.579 & 4.158 & .0006 \\
$\quad$ CPR & & & \\
Age & -7.105 & -2.687 & .0151 \\
\hline
\end{tabular}

Values are mean differences between physician estimates, scored on a 1$100 \mathrm{~mm}$ visual analog scale.

Physicians who reported discussions about life-sustaining treatment participated in qualitative interviews about each case. We completed in-depth interviews for a total of 16 patient cases. The other three cases were excluded from this analysis. In one case, the physician had initially broached the subject with a patient who became upset. The conversation was halted, and the physician never completed the intended discussion concerning withholding treatment. The other two discussions could not be recalled by the physicians.

Ten of the remaining 16 discussions took place in the clinic, five took place in the hospital, and one patient had discussions in both the hospital and clinic. Only three discussions occurred during the study month. Thus, during the study month, physicians talked with their patients about advance directives in only three of 185 patient visits $(1.6 \%)$.

Discussions regarding advance directives were initiated by physicians in only eight of the 16 cases. All but one of these patients were demented, and physicians frequently listed this or quality of life as the reason for initiating the discussion. One physician said she made it a policy to speak with the families of demented patients. The one patient who was not demented was a retired physician, and this apparently played a role in deciding to raise the issue. According to the physician, "I don't know if it would have either come up or stood up in my mind as clearly having come up if he had not been a physician."

"Steep decline," "failing state of health," and other similar phrases describing worsening clinical status also were frequently given as reasons for raising the issue of life-sustaining treatment. Several discussions were initiated because of questions concerning other treatment decisions. For instance, one patient had rectal bleeding, which prompted a discussion (with the family) of whether surgery would be pursued, eventually leading to a discussion about aggressive treatment in general. Another patient had the issue raised after surviving emergency surgery (he chose aggressive treatment, to the surprise of his physician). A third patient refused bypass surgery, and the physician and patient decided together not to pursue repeat angioplasty. At the next clinic visit the physician raised the question of CPR, "because in my mind there was a chance that that scenario could occur in the next year."
Eight discussions were raised by the patient or family; one physician reported a patient who raised the issue at every clinic visit, and another patient was reluctant to have treatment for any condition, including hypertension. Stated the physician, "Almost everything that I recommend results in a major discussion ... (I) raised it in the context of refusing therapy." One patient talked with her physician about life support after her sister had had a prolonged stay in the intensive care unit.

Eleven of 16 discussions involved the patient, three of whom were demented. Of the five discussions that took place only with surrogates, four of the patients were demented; the other was not included because he was "too nervous."

Only two of 16 discussions about life-sustaining treatment resulted in a decision to pursue aggressive therapy. Thirteen patients (or families) decided to withhold life-sustaining treatment, and one patient wanted her physician to make the decision.

\section{DISCUSSION}

Despite a consensus among ethicists that discussions about life-sustaining treatment should take place early, while the patient is competent, discussions occurred in only $10 \%$ of our sample of elderly outpatients, half of whom were considered demented by their physicians. This is considerably less than the proportion of patients who would like to discuss life support options with their physicians. ${ }^{4,11,15}$ Our study confirms that discussions were more likely to occur in older patients and those with worse prognoses. While the quantitative questionnaire scores for quality of life were not associated with discussions about life-sustaining treatment, physicians often mentioned the patient's poor quality of life when asked in interviews why they initiated the discussion. Our interview results show that, even in an outpatient setting, physicians usually do not ask about treatment preferences until the patient is no longer competent to make decisions. In fact, although poor prognosis, and possibly quality of life, were expressed as reasons for initiating discussions, the majority of patients judged by their physicians to have a poor prognosis or quality of life did not have advance directives discussed with them. Often, some clinical event was necessary for the physician to raise the issue, or else the patient or family initiated the discussion. Because the patients were often incompetent, discussions frequently occurred with patients' families. Since families and physicians do not reliably make decisions that patients would have chosen, ${ }^{16,17}$ discussing life-sustaining treatment with families may not result in a decision that the patient would have chosen.

Other investigators have found a low frequency of discussions about advance directives in both inpatient and outpatient settings ${ }^{14,15,18}$ and found prognosis, quality of life, and specific diseases to be associated more frequently with Do Not Resuscitate orders in the inpatient setting. ${ }^{17,19-22}$ Our study found similar patient factors were important in outpatient discussions. Dementia apparently influences physicians to discuss the withholding or withdrawing of treatment, perhaps in- 
dicating the value physicians themselves place on cognitive ability. Clinical events often precipitated discussions about life-sustaining treatment. Possibly, these serve as a reminder or warning that the issue of withholding treatment may arise in the near future. Alternatively, they may provide a convenient launching pad for physicians to initiate difficult discussions in the context of patient care.

Despite strong feelings about life support, patients have not raised the topic with their physicians. ${ }^{4,11,23}$ Despite acknowledging the value of such discussions, physicians often do not raise the topic with their patients until the patient is critically ill and/or incompetent. The reasons for this stalemate remain unclear. Patients may be uncomfortable discussing a topic that requires acknowledging their mortality. They may be uncomfortable in the assertive role of initiator, as they are reluctant to raise other sensitive issues with their physicians. ${ }^{12}$ Uncertainty about how to talk to patients about death, fear of emotionally harming their patients, time constraints and/or lack of reimbursement may inhibit physicians from raising the issue. One author has advocated a separate Medicare fee code to encourage discussions about advance directives. ${ }^{24}$ It may be that physicians simply do not think of talking to patients about this until the issue is brought to the forefront in the context of clinical care or by the patient him/herself. At least for most physicians, at present it is not a part of routine "health care maintenance."

The consequences of patients' and physicians' inability to talk about life supports are deplorable: desired treatments are withheld and unwanted care is administered at great personal and societal cost. More frequent discussions about life-sustaining treatment not only improve the quality of care by respecting patients' treatment goals, they may lead to less unwanted care administered and result in a decline in expenditures! Legally, the importance of premorbid discussions about limiting treatment has never been greater, as documentation of such in the medical record may serve as the "clear and convincing evidence" of the patient's wishes necessary (in some states) to withhold or withdraw lifesustaining treatment since the Cruzan decision.

Since patients admitted to the hospital or nursing home are often too ill or incompetent to make decisions, policies aimed at these institutions (such as the Patient Self-Determination Act of 1990) may not be sufficient. A more suitable site for decision-making about advance directives is the ambulatory care setting. Away from the intimidation and stress of hospitalization, patients can take time to consider the options and discuss them with their loved ones and their primary care provider. Furthermore, this setting is more conducive to relaxed discussion. The time spent with the patient is likely to be less than the time required should the patient later become seriously ill and reflects the patient's authentic preferences rather than a surrogate's imperfect estimate. Treatment wishes, when discussed early with a primary care physician able to interpret an individual's goals and values, may avoid the inflexibility sometimes associated with advance directive documents.

The reported study was intended as a detailed ex- amination of doctor-patient discussions about limiting treatment, and the analysis of associations is limited by its small size, the low frequency of discussions, and the clustering of discussions among a few physicians. Our power to detect smaller differences between patients or physicians, or subtler associations, was limited. In addition, only three of the discussions actually took place during the month of the survey. This may have compromised physicians' recall about the context and content of the discussions. It may be, for instance, that physicians tend to remember more vividly those discussions that are held with surrogates, and this biased our results toward the finding of more discussions in demented patients. Other investigators, however, have also found DNR orders and discussions about life-sustaining treatment to be more frequent for incompetent patients. Although recall bias may have affected other findings about the context and content of discussions, physicians were surprisingly specific in their responses to interview questions about location and preciptating factors. Answers about the timing seemed, not surprisingly, less confident. Recall bias also tends to underestimate the frequency of discussions about life-sustaining treatment; since we did not survey patients or their surrogates we may have underestimated the events. Discussions not recalled by physicians would, presumably, be a less accurate source of information about why physicians raise the issue, and, hence, the study was not designed to identify them.

Unfortunately the original questionnaire did not specifically address the question of dementia, a factor identified by the interviews as influential. This could have been done either with a standard dementia scale or by merely asking the physicians for their assessment of the patient's mental status. Our survey asked for an assessment of competence (which did not correlate with discussions), but this is only a crude proxy for mental status.

Future work in this area could address the issue of dementia as an important factor in prompting physicians to initiate discussions about life-sustaining treatment, and individual physician characteristics and attitudes were not found to be of significance in this study. It would also be interesting to collect data about patient-physician discussions more directly using direct observation and taped conversations. There has been some preliminary work evaluating the efficacy of mechanisms designed to increase the frequency of discussions about limiting treatment in the outpatient setting. ${ }^{25}$ Similar research, evaluating educational, administrative, or incentive programs, may succeed in ameliorating this unfortunate silent stalemate between doctors and patients.

\section{REFERENCES}

1. The Hastings Center. The Hastings Center Guidelines on the Termination of Life-Sustaining Treatment and Care of the Dying. Indianapolis: Indiana University Press, 1987.

2. Meyer DW. Medico-Legal Implications of Death and Dying. Rochester: The Lawyers Co-Operative Publishing Company, 1988.

3. President's Commission for Study of Ethical Problems in Medicine and Biomedical and Behavioral Research. Deciding to Forego Life-Sustaining Treatment: Ethical, Medical and Legal Issues in Treatment Decisions. Washington, DC: United States Printing Office, 1983. 
4. Shmerling RH, Bedell SE, Lilienfeld A et al. Discussing cardiopulmonary resuscitation: A study of elderly outpatients. J Gen Intern Med 1988;3: 317-321.

5. Steinbrook R, Lo B, Moulton J et al. Preferences of homosexual men with AIDS for life-sustaining treatment. N Engl J Med 1986;314:457-460.

6. Havlir D, Brown L, Rousseau GK. Do not resuscitate decisions in a hospitalbased home care program. J Am Geriatr Soc 1989;37:52-54.

7. Bedell SE, Delbanco TL. Choices about cardiopulmonary resuscitation in the hospital. N Engl J Med 1984;310:1089-1093.

8. Lou Harris and Associates. Views of informed consent and decisionmaking: Parallel surveys of physicians and public. In: President's Com mission for the Study of Ethical Problems in Medicine and Biomedical and Behavioral Research. Making Health Care Decisions, Vol. 2. Washington DC: United States Government Printing Office, 1982, pp. 217-263.

9. Kohn M, Monon G. Life prolongation: Views of elderly outpatients and health care professionals. J Am Geriatr Soc 1988;36:840-844.

10. Snow RM, Atwood K. Probable death: Perspective of the elderly. South Med J 1985;78:851-853.

11. Lo B, McLeod GA, Saika G. Patient attitudes to discussing life-sustaining treatment. Arch Int Med 1986;146:1613-1615.

12. Siminoff LA, Fetting JH, Abeloff MD. Doctor-patient communication about breast cancer adjuvant therapy. J Clin Oncol 1989;7:1192-1200.

13. Folstein MF, Luria R. Reliability, validity and clinical application of the visual analogue mood scale. Psychol Med 1973;3:479-486.

14. Scott J, Huskisson EC. Graphic representation of pain. Pain 1977;2: $175-184$.

15. Finucane TE, Shumway JM, Powers RL, $D^{\prime}$ Allesandri RM. Planning with elderly outpatients for contingencies of severe illness. J Gen Intern Med
$1988 ; 3: 322-325$.

16. Uhlmann RF, Pearlmann RA, Coin KC. Physicians' and spouses' predictions of elderly patients' resuscitation preferences. I Gerontol Med Sci $1988 ; 43: 115-121$.

17. Ouslander JG, Tymchuk AJ, Rahbar B. Health care decisions among elderly long-term care residents and their potential proxies. Arch Intern Med 1989; 149:1367-1372

18. Blackwell LJ, Cobb I, Moskowitz MA. Discussions regarding aggressive care with critically ill patients. J Gen Intern Med 1989;4:399-402.

19. Bedell SE, Pelle D, Maher PL et al. Do-Not-Resuscitate orders for critically ill patients in the hospital: How are they used and what is their impact? JAMA 1986;256:233-238.

20. Youngner SJ, Lewandowski W, McClish DK et al. "Do Not Resuscitate" orders: Incidence and implications in a medical intensive care unit. JAMA 1985;253:54-57.

21. Frampton MW, Mayewski RJ. Physicians' and nurses' attitudes toward withholding treatment in a community hospital. J Gen Intern Med 1987, 2:394-399.

22. Pearlman RA, Jonsen $A$. The use of quality-of-life considerations in medical decision-making. J Am Geriatr Soc 1985;33:344-352.

23. Frankl D, Oye RK, Bellamy PE. Attitudes of hospitalized patients toward life support: A survey of 200 medical inpatients. Am J Med 1989;86: 645-648.

24. Murphy DJ. Improving advance directives for healthy older people. J Am Geriatr Soc 1990;38:1251-1256.

25. Sachs GA, Stocking CB, Miles SH. Empowerment of the older patient? A randomized, controlled trial to increase discussion and use of advance directives. J Am Geriatr Soc 1992;40:269-273 